\title{
Electron cloud dynamics in the Cornell Electron Storage Ring Test Accelerator wiggler
}

\author{
C. M. Celata* \\ Cornell University, Ithaca, New York 14853, USA
}

(Received 6 July 2010; published 7 April 2011)

\begin{abstract}
The interference of stray electrons (also called "electron clouds") with accelerator beams is important in modern intense-beam accelerators, especially those with beams of positive charge. In magnetic wigglers, used, for instance, for transverse emittance damping, the intense synchrotron radiation produced by the beam can generate an electron cloud of relatively high density. In this paper the complicated dynamics of electron clouds in wigglers is examined using the example of a wiggler in the Cornell Electron Storage Ring Test Accelerator experiment at the Cornell Electron Storage Ring. Three-dimensional particle-in-cell simulations with the WARP-POSINST computer code show different density and dynamics for the electron cloud at locations near the maxima of the vertical wiggler field when compared to locations near the minima. Dynamics in these regions, the electron cloud distribution vs longitudinal position, and the beam coherent tune shift caused by the wiggler electron cloud will be discussed.
\end{abstract}

DOI: 10.1103/PhysRevSTAB.14.041003

PACS numbers: 29.27.- a, 41.75.Ht

\section{INTRODUCTION}

Stray electrons are formed in high-energy circular accelerators or storage rings when synchrotron radiation from the beam hits the vacuum wall, when lost beam particles strike the wall, or by ionization by the beam of background gas. These "primary electrons" or "photoelectrons" can then produce secondary electrons upon impact with the chamber wall. Secondaries can also strike the wall, producing more secondaries, so that the population of secondaries under some circumstances can outnumber the primary electrons. An "electron cloud" formed in this way can negatively impact beam stability and quality [1]. If the beam particles are of negative charge they tend to repel the electrons, keeping them far from the beam and near the chamber wall, where they can be absorbed. Thus, in negative-particle accelerators the effect of the electron cloud is relatively weak. But for modern high-intensity accelerators with positively charged beams, electron cloud effects often present an important limit to the luminosity [1,2]. In the International Linear Collider (ILC) as presently designed, for example, electron cloud effects are calculated to be important in the positron damping ring, and the expected impact of the electron cloud on the beam and the success of various proposed mitigations will be important in deciding the number and circumference of the positron damping rings. Experiments on the Cornell Electron Storage Ring

\footnotetext{
*Visitor at California Institute of Technology, 1200 E. California Blvd., Pasadena, CA 91125.

Guest at Lawrence Berkeley National Laboratory, 1 Cyclotron Road, Berkeley, CA 94720.

CMCelata@lbl.gov

Published by American Physical Society under the terms of the Creative Commons Attribution 3.0 License. Further distribution of this work must maintain attribution to the author(s) and the published article's title, journal citation, and DOI.
}

(CESR), called the CESR Test Accelerator (CESR-TA) experiments, include as a major component studies of the buildup of the electron cloud, its effect on the beam, and mitigations. Many characteristics make CESR a good test bed for these experiments, particularly its high-intensity beam with small vertical emittance, its flexibility with respect to bunch spacing and bunch train length, the ability to accelerate either positron or electron beams for comparisons, and the presence of high field wigglers, whose design is presently the template for the ILC damping ring wigglers. In this paper we report the results of 3D particle-in-cell simulations of the buildup of the electron cloud in a wiggler located in the L0 straight section of CESR-TA.

The magnetic field of a wiggler is inherently three dimensional, and because the electron cloud buildup is different depending on the local magnetic field structure, and therefore different at different longitudinal locations, the electric fields of the electrons are also three dimensional. Additional complexity comes from the pinching of the electron cloud as a positively charged beam passes through it. This gives longitudinal structure to the electron cloud, creating longitudinal as well as transverse electric forces. For all of these reasons the dynamics of the electron cloud in a wiggler is three dimensional by its nature, so fully three-dimensional computer calculations are used here to examine the cloud buildup and behavior.

Previous studies of the electron cloud in wigglers have employed simulations with both two- and threedimensional models. The simulations in Refs. [3-6] used models with full three-dimensional wiggler field models and three-dimensional electron dynamics except for the applied electron space charge forces, which were 2D. However, though the simulations calculated electron cloud density in all regions of the wiggler, the results for cloud buildup show only the time development of the total electron density integrated over the length of the wiggler. The 
distribution of electron density as a function of distance along the length of the wiggler is not shown. The papers also contain no discussion of the electron dynamics except in areas of strong vertical field, where the system is very like a dipole and therefore essentially two dimensional. In particular, they do not address the details of the electron motion near the points in the wiggler where the vertical magnetic field, $B_{y}$, is zero. Finding the electron distribution as a function of distance along the wiggler and understanding the electron dynamics, especially in the regions of low vertical field, are prominent parts of this report.

A few studies have simulated the wiggler electron cloud buildup in three dimensions, including full 3D treatment of the electron space charge [7-10]. In 2007 the author first demonstrated that electrons cross field lines near $z$ locations in the wiggler where $B_{y}=0$ [7]. This behavior is a major part of the dynamics of electrons in the wiggler, and is discussed and explained below in detail. Other papers in $2007[8,9]$ calculated the buildup of the wiggler electron cloud but did not mention seeing these electrons or explore the electron dynamics in the region of minimal $B_{y}$. Rather, electron dynamics was described only in areas of fairly strong vertical magnetic field. All of the material in the present report, including explanations and proof of the reasons for the above-mentioned phenomena and the long-lived nature of the electrons crossing field lines near minimal $B_{y} z$ locations, was presented by the author at electron cloud meetings of the CESR-TA collaboration between February 9 and September 23 of 2009. Subsequently, after several suggestions by the author, a member of the collaboration, Wang, was able to confirm the findings near $B_{y}=0$ with the $3 \mathrm{D}$ simulation code CLOUDLAND, and those results were published in the proceedings of IPAC10 [10]. Thus, though work has been done previously to calculate the electron cloud buildup in wigglers, the present report is the first to display and investigate in detail electron dynamics in parts of the wiggler where the field is not essentially vertical.

This paper is organized in the following fashion. In Sec. II the computational model is explained in detail. Section III gives results for buildup of the electron cloud in various areas of the wiggler. In Sec. IV calculations of the beam coherent tune shift due to the electron cloud are discussed. Finally, Sec. V gives a conclusions and a summary of the paper.

\section{THE COMPUTATIONAL MODEL AND PARAMETERS}

Because of the three-dimensional character of both the wiggler magnetic field and the interactions among electrons, the 3D particle-in-cell (PIC) computer code WARPPOSINST [11] was used to investigate the electron buildup and dynamics. WARP-POSINST uses the photoelectron and secondary electron models of the POSINST code [12-14] to generate the electrons which appear due to the impact of synchrotron radiation produced by the beam on the vacuum wall and to the electron impact at the walls. The electron and beam dynamics are then followed using the capabilities of the WARP PIC and diagnostic modules. Table I gives

TABLE I. Simulation parameters.

\begin{tabular}{ll}
\hline \hline Quantity & \\
\hline Positrons per beam bunch, $N_{b}$ & $6.4 \times 10^{9}$ \\
Positron energy & $2.085 \mathrm{GeV}$ \\
Bunch spacing, $\tau_{b}$ & $14 \mathrm{~ns}$ \\
$\sigma_{x}$ (beam) & $460 \mu \mathrm{m}$ \\
$\sigma_{y}$ (beam) & $60 \mu \mathrm{m}$ \\
$\sigma_{z}$ (beam) & $8.8 \mathrm{~mm}$ \\
Maximum wiggler field & $1.9 \mathrm{~T}$ \\
Photons emitted per positron per meter & 2.16 \\
Photon reflectivity & 0.2 \\
Quantum efficiency & 0.1 \\
Peak secondary electron yield at normal & 1.8 \\
incidence & \\
Energy at peak secondary electron yield at & $276 \mathrm{eV}$ \\
normal incidence & \\
Vacuum pipe radii (elliptical pipe, $x, y$ radii) & $4.5,2.2 \mathrm{~cm}$ \\
PIC spatial grid cell size $(x, y, z)$ & $0.71 \times 0.71 \times 6.2 \mathrm{~mm}$ or, when dynamics \\
& allow, $1.42 \times 1.42 \times 6.2 \mathrm{~mm}$ \\
Time Step & $1.57 \times 10^{-12} \mathrm{~s}$ \\
Real electrons represented by each & $1490 \mathrm{except}$ for tune shift calculations, where \\
simulation macroparticle & the number is initially 37 but increases as the \\
& simulation progresses \\
\hline \hline
\end{tabular}


numerical and physical parameters used for the simulations described in this paper.

Though WARP-POSINST is a fully self-consistent PIC code, and therefore could be used to follow the complete dynamics of both beam and electrons, certain simplifications have been made here in order to speed up the CPUintensive calculations. The beam distribution function was not allowed to evolve in time. This is thought to be a reasonable assumption during the short (here, $<$ a few $\mu$ s) buildup time for the cloud. Instead, the beam field was represented by the $2 \mathrm{D}$ Bassetti-Erskine field, which is appropriate for a beam with a Gaussian distribution function in all three dimensions traveling at essentially the speed of light. To simplify the understanding of the dynamics and for better comparison with previous calculations of dynamics in a dipole, the beam followed a trajectory straight down the center of the elliptical beam pipe-i.e., the wiggle of the centroid was ignored. The effect of this approximation is small since offset of the beam centroid by a small amount has been seen in simulations to simply move the center of the electron cloud without significantly affecting the cloud buildup. Because of the relatively low velocity of the vast majority of the electrons, the magnetic field of the beam was neglected. Full 3D electron dynamics, including interactions of electrons with other electrons, were included. The image forces of both the beam and the electrons were included in the calculation. The simulation coordinate system was defined such that the beam travels in the $+z$ direction.

Cornell results from a calculation of the magnetic field of the wiggler using the OPERA code were used to provide a tabular representation of the magnetic field as input to WARP, which linearly interpolates the field to the position of each particle.

In CESR-TA, as in the ILC, the number of electrons formed by ionization of background gas is negligible, so the electrons which are born and followed in the simulations result from the impact of either synchrotron radiation or electrons on the vacuum chamber wall. Though most of the CESR vacuum chamber is aluminum, the wiggler vacuum chamber is copper, and this was reflected in the choice of the coefficients for unprocessed copper for the POSINST secondary emission model in the calculations. In the simulations the vacuum chamber shape was elliptical. The true shape of the wiggler chamber cross section is shown in Fig. 1 along with the simulation chamber cross section. An important parameter for the calculation is the number of synchrotron photons produced per beam particle per meter. These photons come from upstream, since the radiation cone is narrow, and they are assumed in the calculation to travel concurrently with the beam. Since calculations of the photon intensity in the wiggler are in a preliminary stage, an approximate value of the intensity believed to be present in the wiggler has been used (see

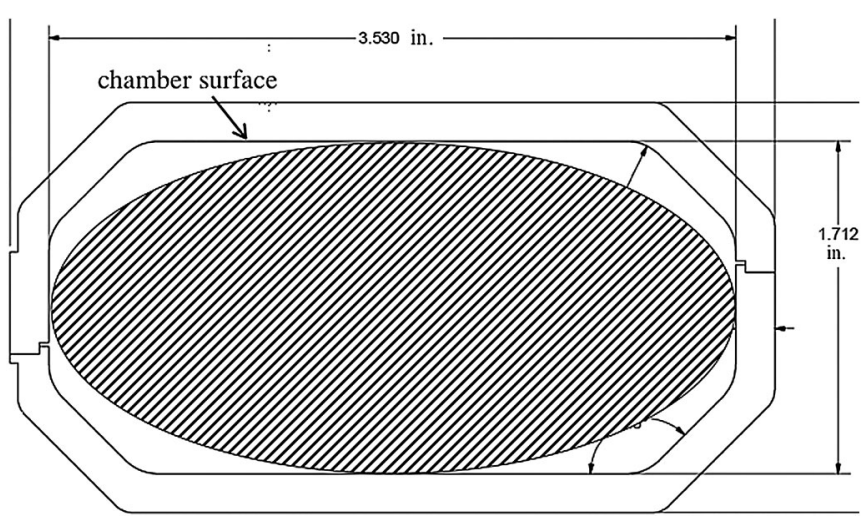

FIG. 1. Wiggler vacuum chamber with elliptical vacuum chamber used in simulations superimposed (crosshatched).

Table I), and the same value has been used at all $z$. The value of the photon reflectivity used is also approximate, but the same value has been obtained at Cornell from multivariable fits of simulations to experimental data taken with a retarding field analyzer (RFA) located in the wiggler at the $z$ position of maximal magnetic field. The reflectivity in the table represents the proportion of photoelectrons which reflect from the positions near the midplane ( $x= \pm R$, where $R$ is the chamber major radius, and $y=0$ ), where the direct radiation cone impacts the vacuum chamber. In the simulation these photons are assumed to create photoelectrons which are distributed uniformly (per unit arclength) along the rest of the perimeter of the vacuum chamber cross section.

Numerical parameters such as the spatial resolution of the PIC grid and the time step were varied until they were clearly in a regime where enhancing resolution (in time or space) had a negligible effect on the result. The time step necessary to resolve the cyclotron period at the peak wiggler field is exceedingly small $\left(1.6 \times 10^{-12} \mathrm{~s}\right)$, and since all electrons have the same time step in the WARP-POSINST runs, this small time step resulted in long computer runs, especially for coherent tune shift calculations, where very good particle statistics are necessary. Tune shift calculations for 45 bunches passing through the $40-\mathrm{cm}$ wiggler period required 405 hours of wallclock time (for each of two runs necessary to calculate the tune shift) using 16 processors on the Lawrencium computer cluster at LBNL. In contrast, the cloud buildup calculation for the same case (but with fewer macroparticles needed for good statistics) required only 21 hours.

Resonances of the electron cyclotron motion with the bunch passage frequency will occur where the magnetic field is such that the cyclotron frequency is an integral multiple of the bunch frequency [15]. The magnitude of this effect on the electrons is only non-negligible at low magnetic fields, where the time for a bunch to pass an electron is much less than the cyclotron period [15]. The $z$ resolution of the electron space charge field in the 
calculations shown in this report is not sufficient to resolve individual cyclotron resonances, which would require resolution to at least $\sim 10 \mu \mathrm{m}$. Present resolution is $0.71 \mathrm{~mm}$ in $x$ and $y$ and $6.2 \mathrm{~mm}$ in $z$. Note, however, that all electrons will feel the correct field from the beam, which is applied using the Bassetti-Erskine formula, and therefore those within resonances will acquire the appropriate extra velocity from the resonance. Indeed, increased electron energy is seen in the vicinity of the resonances in these calculations. What is not resolved correctly is any variation of the electron space charge field on a scale below the dimensions just quoted. However, such an effect would be expected to be smeared out quickly anyway as electrons move along field lines since the resonances occur on such a fine spatial scale.

Simulations of the wiggler were done by simulating one period of the wiggler near its longitudinal center using periodic boundary conditions in $z$ for both the fields and the particles so that any particle, which, for instance, exited the system at the high- $z$ boundary immediately entered at the same $x$ and $y$ with the same velocity at the low- $z$ boundary. In some cases shorter sections of the wiggler were simulated, as noted below.

\section{ELECTRON CLOUD BUILDUP AND ITS VARIATION WITH $z$}

\section{A. Orbits of electrons for $\boldsymbol{Z}$ near the maximal $\boldsymbol{B}_{\boldsymbol{y}}$}

The buildup of the electron cloud in a dipole has been studied in considerable detail by others. It is well known that, for sufficiently high bunch currents, in a dipole field two vertical stripes of electron density, one on each side of the vertical $(y)$ axis, occur at $x$ locations where the electrons are within a certain distance range from the beam when the bunch appears. At this distance the beam kick produces electron energies near the peak of the secondary emission yield function [16-18]. If the bunch current of the beam is reduced the stripes will move closer to the $y$ axis until they merge. At $z$ locations very near the maximum vertical field of the wiggler, where the magnetic field geometry is close to that of a dipole, our simulations show the electron cloud buildup for the wiggler to be identical in buildup time and electron density to calculations done with an ideal dipole field. For the parameters of Table I the cloud buildup is shown in Fig. 2, and the density in the $x-y$ plane (integrated over a $z$ length of $6.24 \mathrm{~mm}$ centered at the $z$ of maximal $B_{y}$ ) in Fig. 3. Figure 2 shows the density of the primary electrons and the secondaries separately, as well as the total. From this, one can see that though the population of primaries is significant, the secondaries dominate. Note that Fig. 2 shows the "average density," which for the purposes of this paper is the density averaged over the complete vacuum chamber volume included in the calculation. For Fig. 2, for instance, the $z$ range over which the average is performed is $6.24 \mathrm{~mm}$ centered at the $z$ of the maximum in $B_{y}$. Good agreement

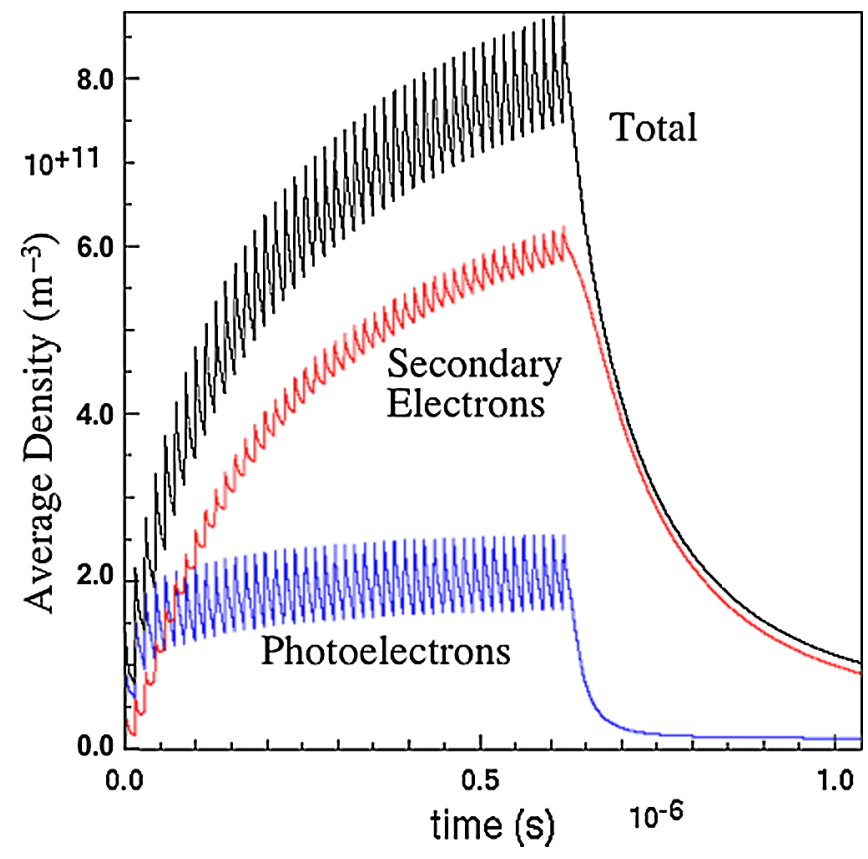

FIG. 2. Buildup of electron density vs time for a 45-bunch train for a $z$ length of $6.24 \mathrm{~mm}$ centered at the maximum of $B_{y}$ in the wiggler. Each bunch passage causes a spike in the density.

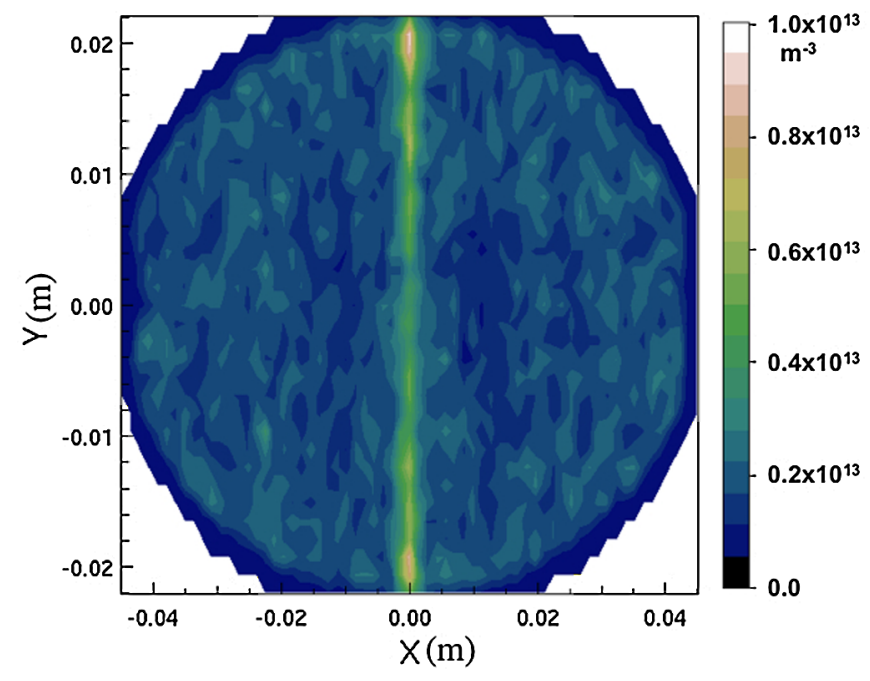

FIG. 3. Electron density in the $x-y$ plane for all electrons with $z$ within $3.134 \mathrm{~mm}$ of the maximum of $B_{y}$. Snapshot in time at $t=626$ ns, i.e., just after the 45 th bunch.

has been obtained between retarding field analyzer measurements done at the $z$ location of the maximum $B_{y}$ in the wiggler and simulations done for an ideal dipole field (and therefore also with these 3D simulations), though interesting artifacts caused by the diagnostic itself also need to be taken into account in analyzing the data [19]. 


\section{B. Orbits of electrons near $B_{y}=0$ and in intermediate regions}

\section{Motion in the $x$ direction}

A very different density pattern is seen near the $z$ locations where $B_{y}=0$. Figure 4 shows the electron density in the $x-y$ plane at two different times for a $z$ length of wiggler of $6.24 \mathrm{~mm}$ centered at the $z$ where $B_{y}=0$. The $z$ where $B_{y}=0$ will be referred to below as the position of the "field null." As illustrated in Fig. 4, electrons slowly move across the chamber near the midplane $(y=0)$ of the $x-y$ plane, reaching the center at about the time of passage of bunch 45 .

Because the magnetic field is mainly longitudinal in the $z$ range near the field null, the prevailing opinion before this work was begun was that electrons would be unable to cross field lines and move away from the chamber wall. However, the magnetic field in this region is not that of an ideal solenoid. It has nonzero curvature and gradient. Therefore the trajectory of the center of the gyro-orbit of each electron (also called the orbit "guiding center") will experience the well-known "gradient and curvature $B$ " drift. This adds to the velocity the guiding center would have without this gradient and curvature of the field a velocity, $v_{d}$, equal to

$$
v_{d}=\frac{m}{e} \frac{\nabla|B| \times \vec{B}}{|B|^{3}}\left(v_{\|}^{2}+\frac{1}{2} v_{\perp}^{2}\right)
$$

where $B$ is the magnetic field, $m$ and $-e$ are the mass and charge of the electron, and $v_{\|}$and $v_{\perp}$ are the velocities of the electron parallel and perpendicular to the magnetic field, respectively.

Records of single particle orbits in the simulation were compared to Eq. (1). The results are shown for some representative electrons in Fig. 5. It is clear that the cross-field motion is explained by the gradient and curvature $B$ drift of the guiding center. This is further confirmed by the fact that replacing the wiggler field by a purely longitudinal magnetic field causes the drift in the $x$ direction to disappear. Moreover, removing the electric field of the beam or the space charge field of the electrons from the simulation has no effect on the particle drift.

The configuration of the electron density in the $x-y$ plane near the field null, as shown in the plot in Fig. 4, is striking. Electrons moving from the right (high positive $x$ ) do not appear at $y=0$, and near $y=0$ their velocity increases as $y$ increases (electrons closest to the $y$ axis are at larger $y$ ). There is no motion obvious for electrons at high $|y|$, away from the midplane. From Fig. 6, one can see that the electrons coming from the left (from negative $x$ to positive $x$ ) in Fig. 4 are not all at the same $z$, but like those coming from the right, form a "forklike" pattern, with negligible electrons at $z=0.2 \mathrm{~m}$ (the $z$ of the field null) and the same pattern of higher velocity at higher $z$ and no electrons moving across in $x$ at higher $|z|$. To explain this pattern, one must refer to the field geometry as shown in Fig. 7. $B_{x}$ and the variations of the field with $x$ are essentially negligible, so it is enough to show, as in the figure, the magnetic field lines at $x=0$ in the $y-z$ plane. There are four different field line geometries in Fig. 7, separated by diagonal separatrices. On the right and left are lines with radii of curvature which are perpendicular to those of the field lines in the top and bottom quadrants. From Eq. (1), one can see that the $x$ drift of electrons in the right and left quadrants is toward $+x$, whereas those in the top and bottom quadrants drift toward $-x$. Therefore in Fig. 4 the electrons coming toward the vacuum chamber center from the left wall (i.e., from negative $x$ to positive) are from the right and left
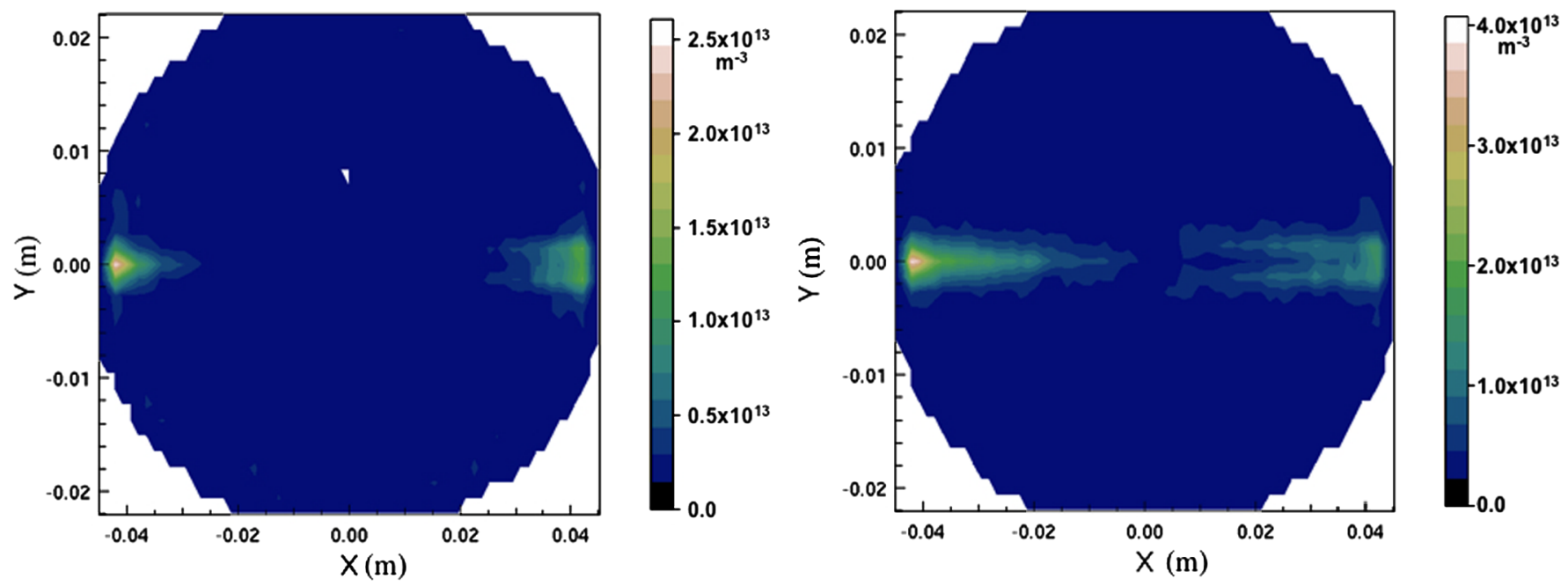

FIG. 4. Density in $m^{-3}$ in the $x-y$ plane for all electrons with $z$ within $3.134 \mathrm{~mm}$ of the $z$ at which $B_{y}=0$. Snapshots in time just before the arrival of bunches 11 (on left) and 45 (on right). 

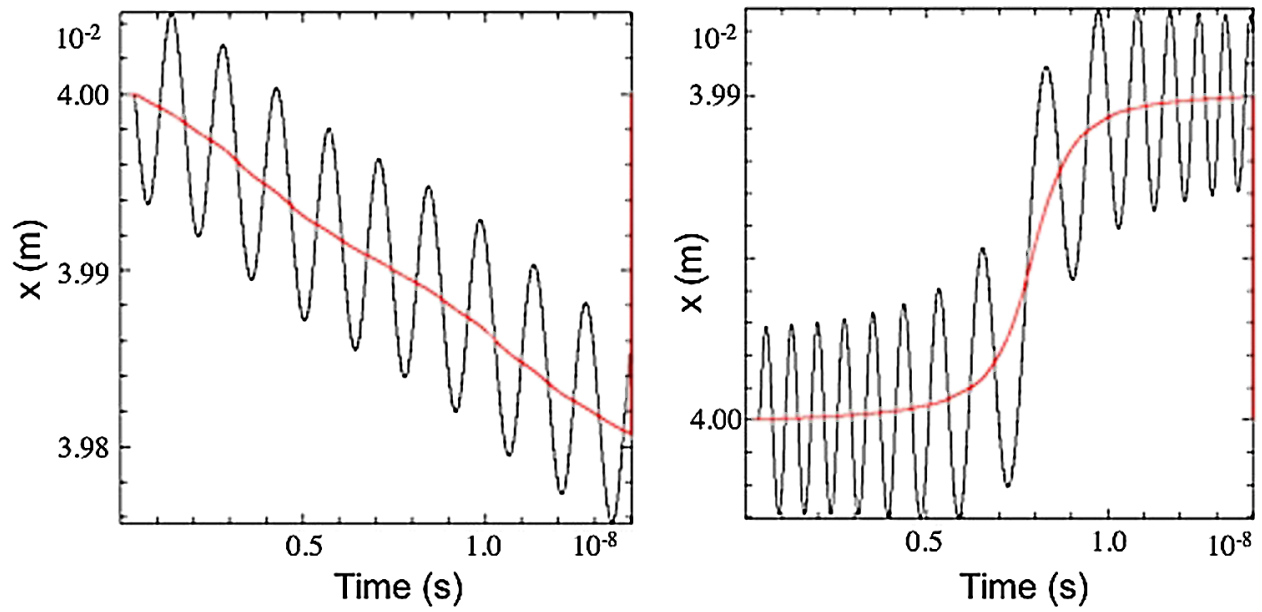

FIG. 5. Drift in the $x$ direction for two randomly chosen electrons near the $z$ where $B_{y}=0$. $x$ vs $t$ is shown for the electron (oscillating lines) as well as the path of the center of the orbit as given by Eq. (1) (smooth lines through center of oscillation).

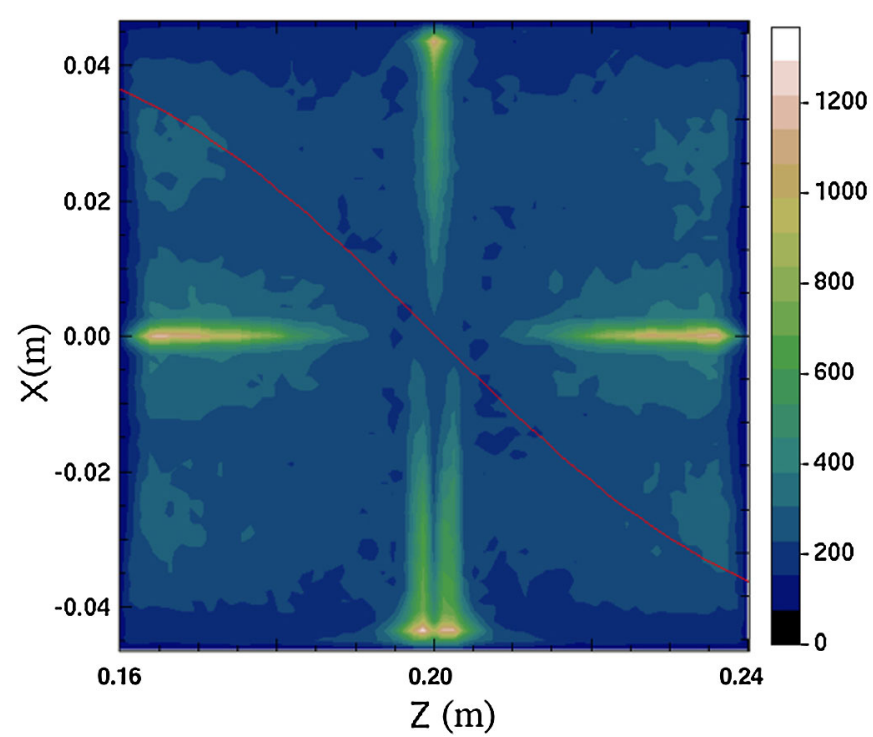

FIG. 6. Color contour plot of electron density in the $x-z$ plane (density is integrated over $y$ ) for a simulation near the field null, which is at $z=0.2 \mathrm{~m}$. Snapshot at $616 \mathrm{~ns}$, i.e., just before the 45 th beam bunch. Color scale is linear (see scale to right) but units are arbitrary. Curve overlaying the plot is proportional to $B_{y}$.

quadrants (this is confirmed by the simulation), and those coming from the right are traveling along field lines in the top and bottom quadrants. In each case there are no electrons in the region near the separatrix. Along the separatrices $\nabla|\mathbf{B}|$ is parallel to $\mathbf{B}$, giving a zero $x$ drift velocity. So approaching the separatrices, as mentioned above, we see the $x$ drift velocity of the electrons decreasing, and finally, at the separatrix, going to zero. This accounts for the fork structure. As we move away to higher $|y|$ at a given $z$, the value of $|B|$ increases, and, as shown in Eq. (1), $v_{d}$ will decrease quickly. So in Fig. 4, which is a snapshot near

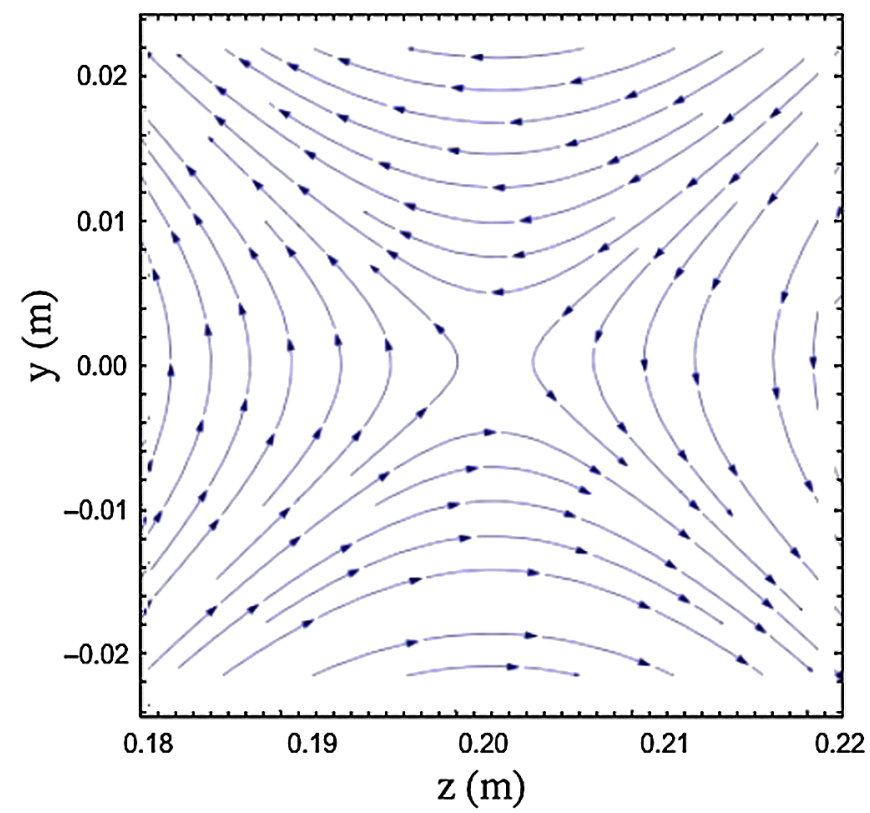

FIG. 7. Magnetic field lines in the $y-z$ plane in the vicinity of the field null, which is at $z=0.2 \mathrm{~m} . x=0$.

the field null, we do not see the $x$ drift of the electrons (on this time scale) except near the midplane $(y=0)$. Likewise, if we move away from the field null in $z$ the value of $|B|$ increases, so that the $x$ motion is not seen near the "dipolelike" sections of the wiggler (see, for instance, Fig. 3).

\section{Motion in the $y$ - $z$ plane}

Along with the slow grad/curvature- $B$ drift of the guiding center in the $x$ direction, the electrons will execute the usual spiraling along field lines. The magnitude of $B$ changes along the field lines, increasing as the electron 


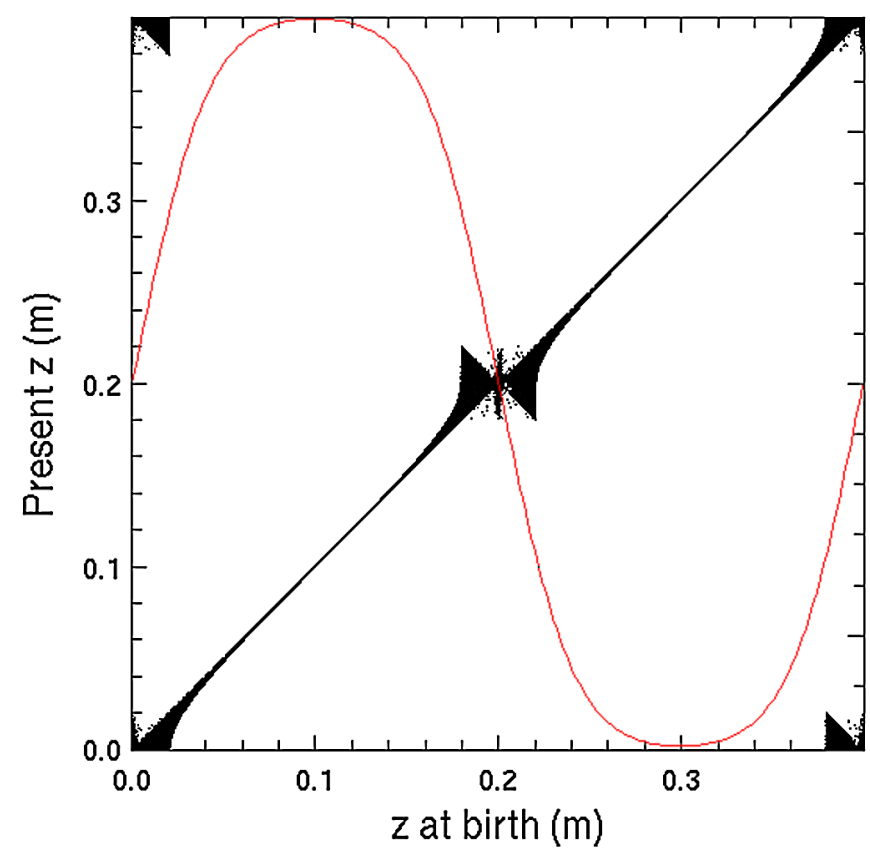

FIG. 8. Movement in $z$ of macroelectrons in the simulation. Each dot represents one macroparticle. Snapshot at $t=701 \mathrm{~ns}$. The field nulls are at $z=0.0,0.2,0.4 \mathrm{~m}$. The sinusoidal-like line shows $B_{y}$ vs $z$. Note that electrons in the upper left and lower right of the diagram are a result of the periodic boundary conditions in $z$ and should be considered as a continuation of the density at the opposite $z$ edge of the plot. moves away from $y=0$ (for field lines at the right and left of Fig. 7) or $z=0$ (for field lines in the top and bottom quarters of Fig. 7). Thus, as is well known (see any basic plasma physics text), as the electron moves along the field line away from the field null its velocity parallel to the field will decrease until, if the magnetic field strength is high enough along its path, $v_{\|}$goes to zero and the electron turns around before it hits the vacuum chamber wall, "mirroring" back and forth along the field line. This mirroring is seen in the electron orbits in the simulations. Thus, electrons near the field null can be trapped on field lines and remain in the system for long times. In Fig. 8, one can see the extent to which electrons move in $z$ in the simulation. This graph does not change in shape for the rest of the simulation run, though some electrons far from the field null hit the wall and leave the simulation. The mirroring effect quantitatively explains the range of $z$ motion seen in this figure for electrons born near the field null, except for those extremely close to the separatrices.

The mirror trapping results in electrons for $z$ near the field null remaining in the chamber well after the last bunch passage, as seen in Fig. 9 (average electron density in the wiggler vs time) and Fig. 10 (average density within $1 \mathrm{~mm}$ of the beam axis vs time). As shown there, this longevity of the cloud does not occur in the sections of the wiggler which are essentially dipolelike. Note also that the density of electrons near the beam at the field null (Fig. 10) is comparable at its peak density to the density
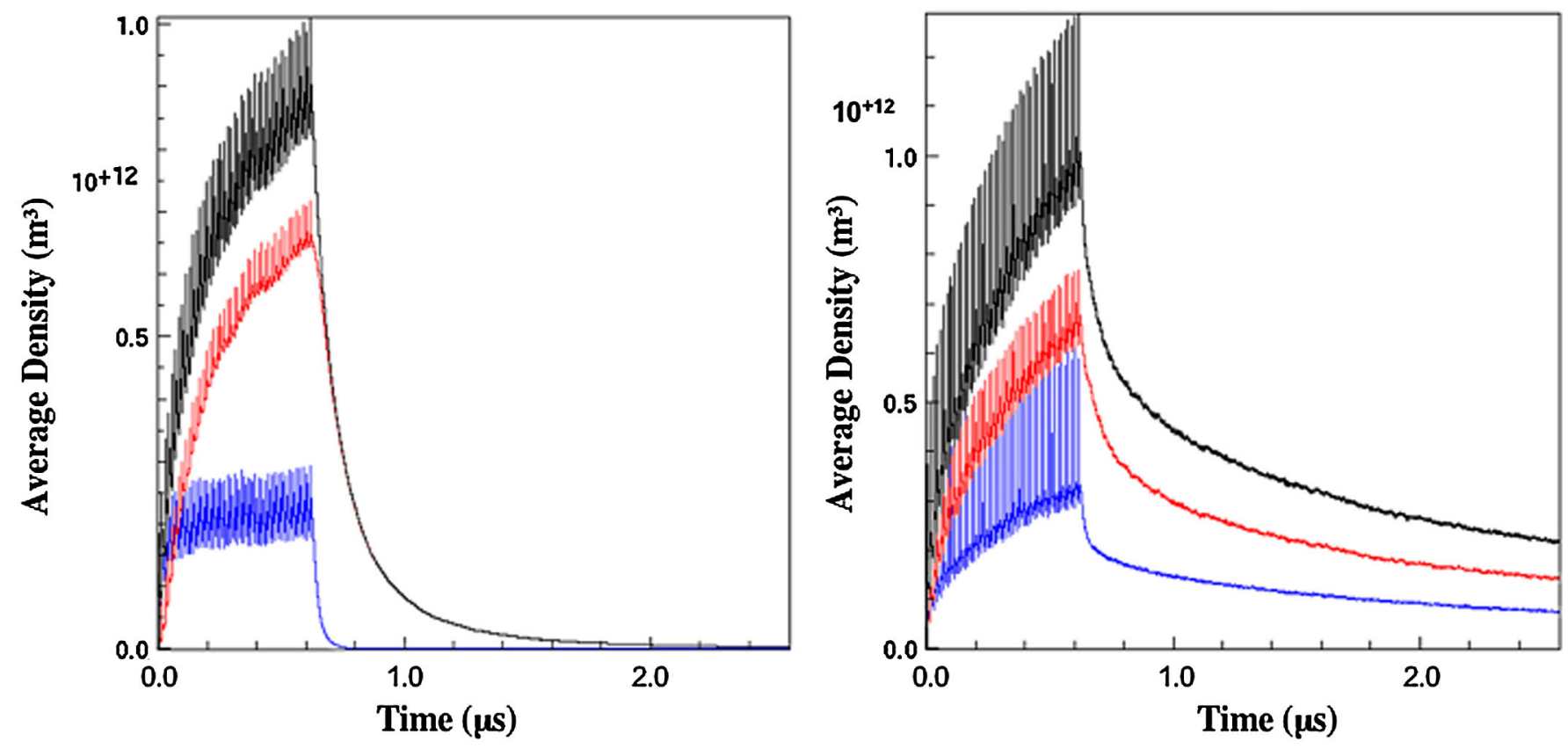

FIG. 9. Buildup and decay of electron density vs time for $z$ within $3.134 \mathrm{~mm}$ of the maximum of $B_{y}$ in the wiggler (left) and $z$ at $B_{y}=0$ (right) for a 45-bunch train. In each case the bottom curve is photoelectrons, middle curve is secondary electrons, and top curve is the total. 

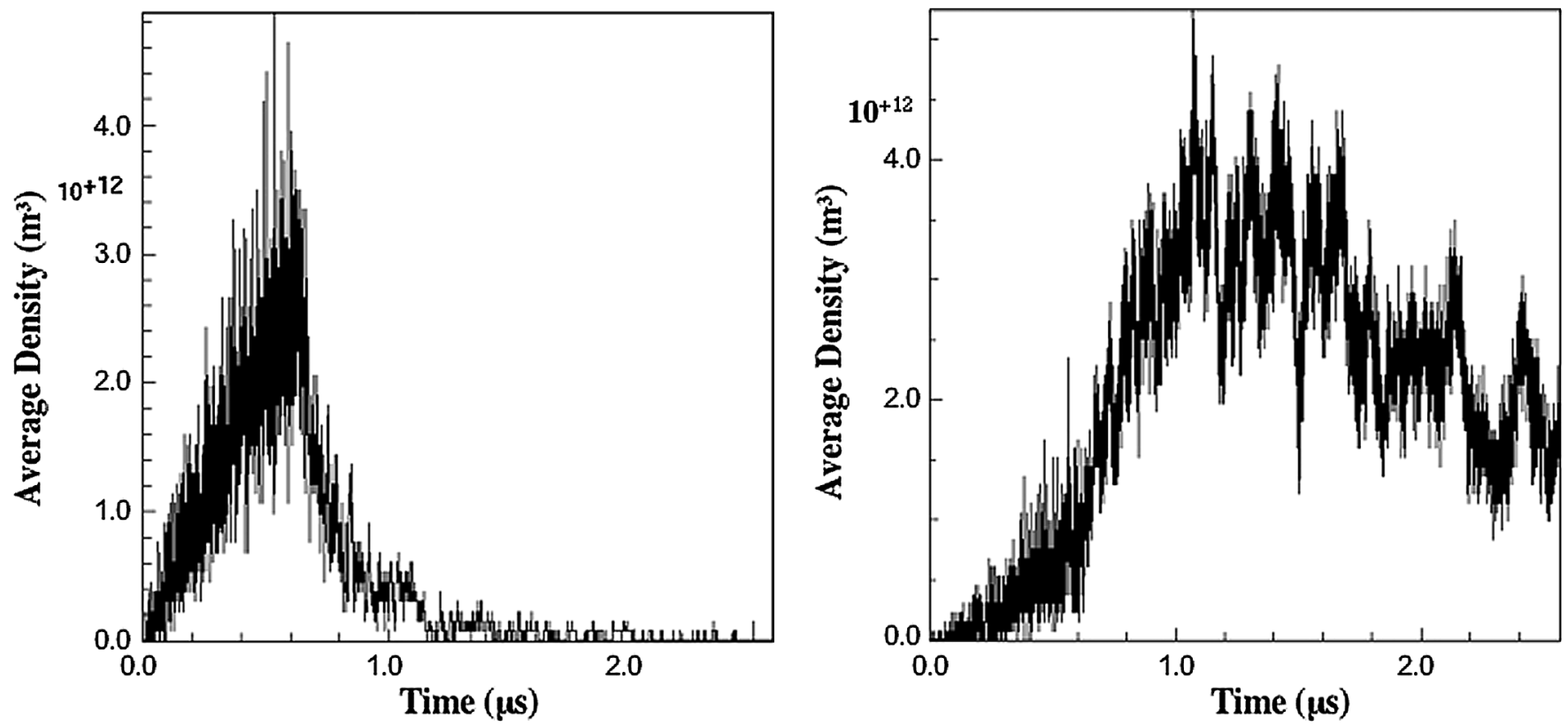

FIG. 10. Average electron density vs time within $1 \mathrm{~mm}$ of the chamber axis $(x=y=0)$ vs time for $z$ within 3.134 mm of the maximum of $B_{y}$ in the wiggler (left) and $z$ at $B_{y}=0$ (right) for a 45-bunch train. Fluctuations are due to macroparticle statistics within this small volume.

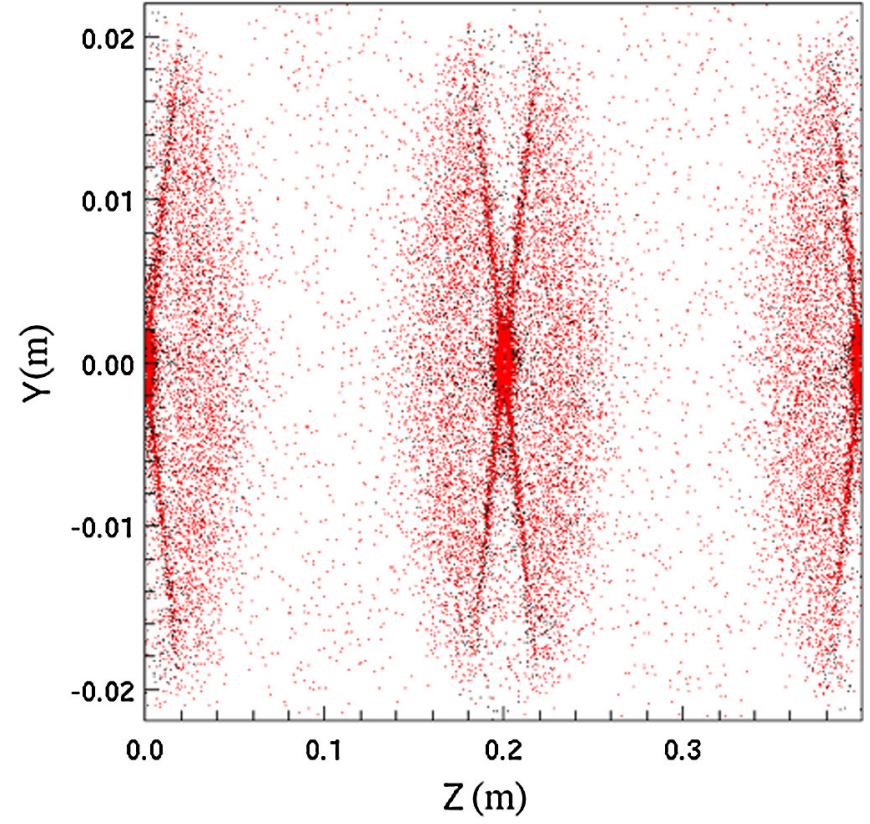

FIG. 11. Macroparticle distribution in the $y-z$ plane at $t=2.56$ microseconds. Field nulls are at $z=0.0,0.2,0.4 \mathrm{~m}$, and maxima of $B_{y}$ are at $z=0.1,0.3 \mathrm{~m}$. Red dots are secondary macroelectrons, black are primary macroelectrons.

seen near the $z$ locations of maximum vertical field, though it takes longer to build to this density. Figure 11 shows the location of this long-lasting density in the $y-z$ plane. Very few electrons are seen for field lines not passing close to $(y, z)=(0,0.2 \mathrm{~m})$, presumably because near the field null, where these long-lasting electrons originate, the high

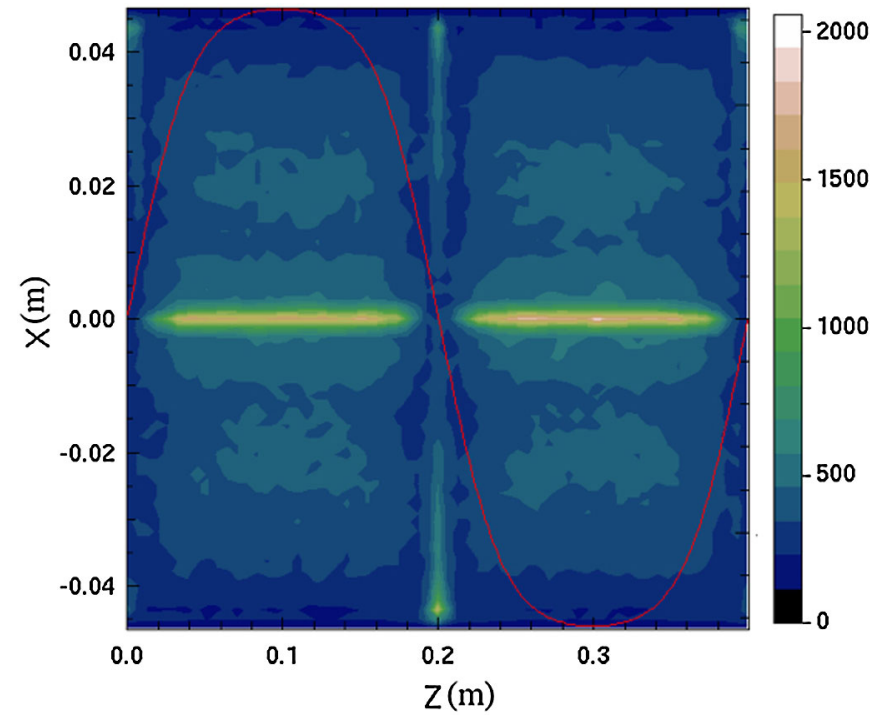

FIG. 12. Electron density in the $x-y$ plane at $t=616 \mathrm{~ns}$, i.e., just before the 45 th bunch passage. The sinusoidal line shows the magnitude of $B_{y}$ vs $z$ at $x=0$. Color bar units are arbitrary but absolute densities can be seen in Figs. 3 and 6 .

density of electrons is in a small range of $y$ near the $x$ axis, as seen in Fig. 4. Another reason could be that the mirror ratio (ratio of the maximum of $|B|$ to its minimum) on the field lines is lower for these field lines. Therefore electrons with high enough $v_{\|} / v_{\perp}$ will not be trapped there and will escape to hit the walls. Figure 12 displays the electron density in the $x-y$ plane near the end of the bunch train. This graph gives another indication of the $z$ ranges of 
the wiggler in which the electron density is like that of a dipole (stripe near $x=0$ ) and those where $z$ is close enough to the field null that $|B|$ is small enough for effective gradient/curvature $B$ drift, giving electron density spread across all $x$.

The fact that electrons stay so long in the chamber suggests that the cloud density from one bunch train might affect succeeding bunch trains, even with what had been considered a significant gap between trains. The electron cloud density near the field null also might grow as suc- cessive bunch trains pass and add to the density left in the chamber. Figure 13 shows the cloud density averaged over the chamber volume for 45-bunch trains with a 15-bunch gap between trains. Note that the bunch spacing here, $14 \mathrm{~ns}$, is much larger than that of the International Linear Collider, which presently is designed for $3 \mathrm{~ns}$ bunch spacing. The average density is shown in the figure for the entire wiggler period [Fig. 13(a)], and for a narrow range of $z(6.3 \mathrm{~mm})$ centered at the maximum of $B_{y}$ [Fig. 13(b)] and for the same range centered at the field null [Fig. 13(c)].
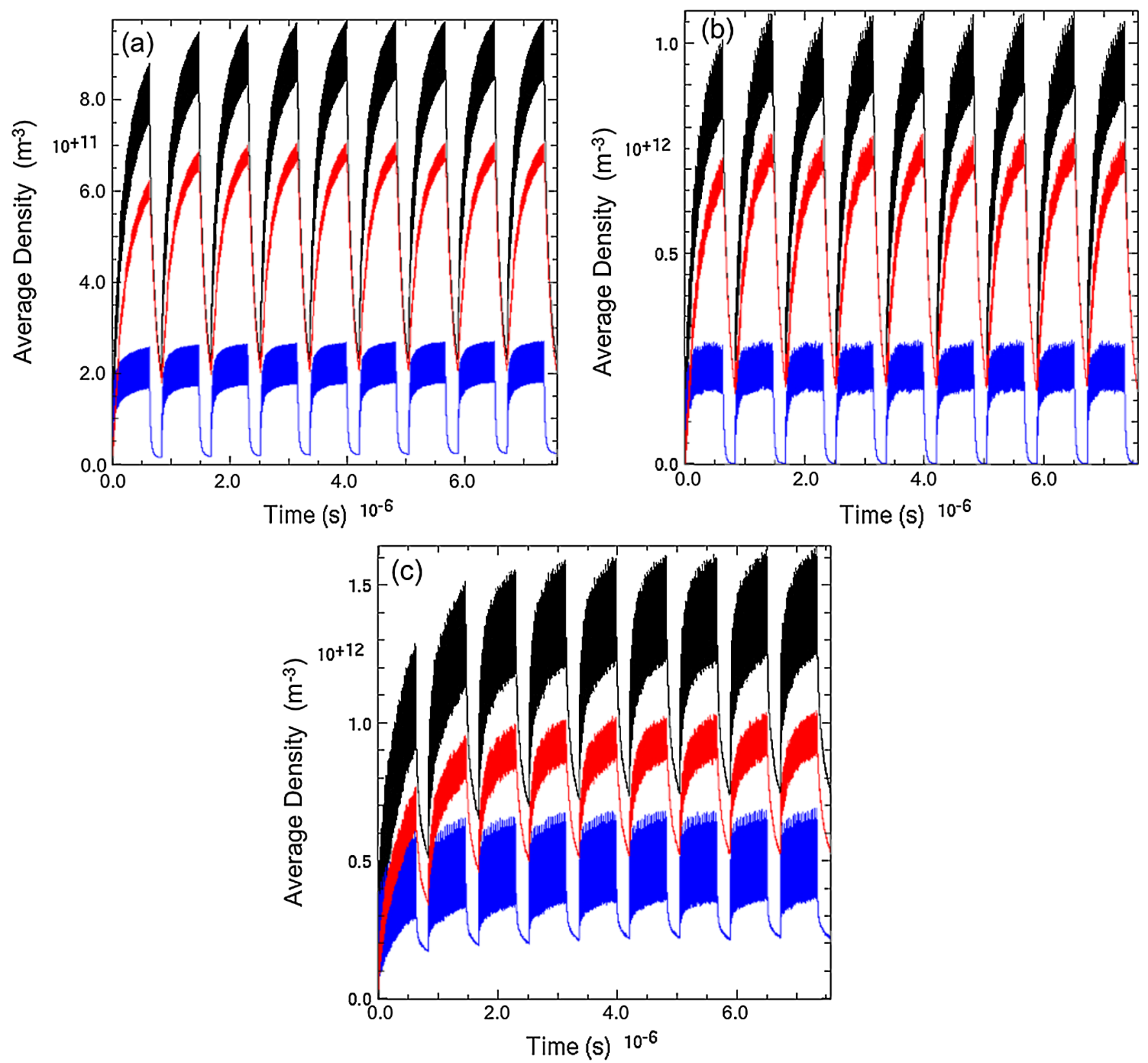

FIG. 13. Average electron density vs time created by nine 45-bunch trains separated by 15-bunch gaps. The lowest curve is photoelectrons, the middle is secondaries, and the top curve is the total. (a) Whole wiggler period, (b) averaged over $6.24 \mathrm{~mm}$ in $z$ centered at a position of maximum $B_{y}$, (c) averaged over $6.24 \mathrm{~mm}$ in $z$ centered at a field null. 


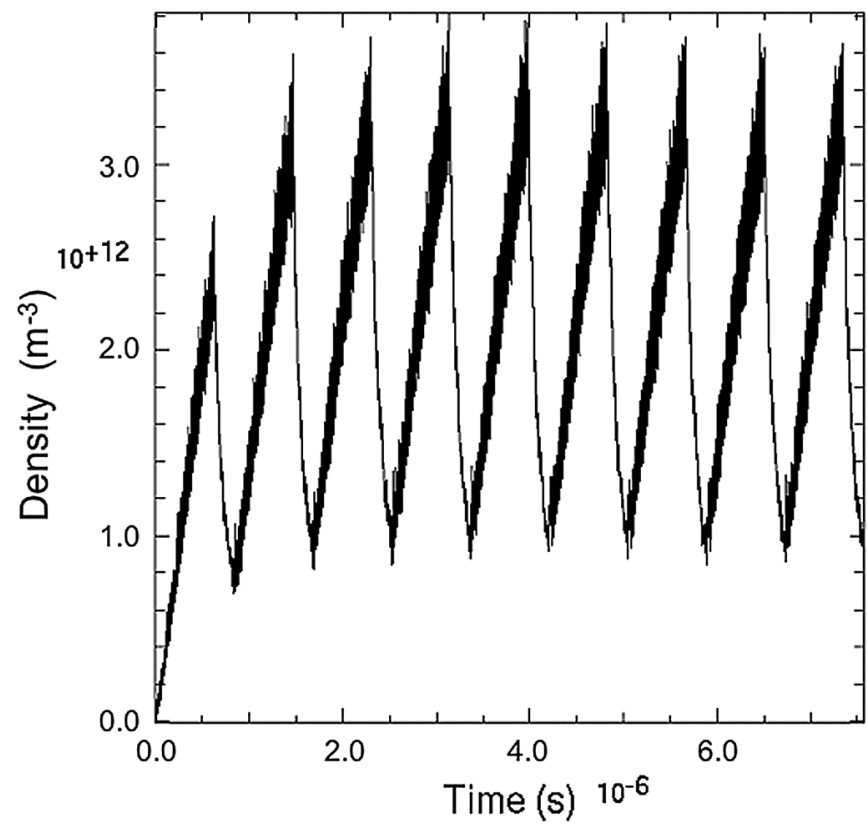

FIG. 14. Density averaged over a cylinder of radius $1 \mathrm{~mm}$ concentric with the chamber axis with a length extending through the entire wiggler period. Nine trains of 45 bunches separated by 15-bunch gaps.

Figure 14 shows the density within a 1-mm-radius cylinder concentric with the chamber axis-i.e., this figure shows the density near the beam. From these figures one can see that, with this bunch spacing, considerable electron cloud lingers through the gaps between bunches. A higher percentage of electrons near the field null remains through the gap, but for this bunch spacing electrons also are still present near the maximum $B_{y}$ locations at the end of the gaps (cf. Fig. 9). This remanent cloud can be expected to have an effect on subsequent trains. The electron density due to the first train is seen to significantly enhance the density present for the passage of the second train. However, the density of the cloud does not increase significantly from train to train after the second or third train. Presumably this is because the cloud is "saturating"—at this point space charge is a dominant feature of the dynamics, causing losses due to wall collisions to balance electron production. The cloud near the field null takes more trains to build to saturation than the cloud near the $B_{y}$ maxima.

\section{Resonances between the beam and electron cyclotron motion}

As mentioned above, the simulations discussed in this paper do not have the resolution in the $z$ coordinate to resolve the space charge field of the electrons on the scale required to resolve the resonances of Ref. [15]. However, the electron space charge is a minor perturbation during much of the cloud buildup, and motion of the electrons in $z$ would be expected to smear any variations in the $z$ direc-

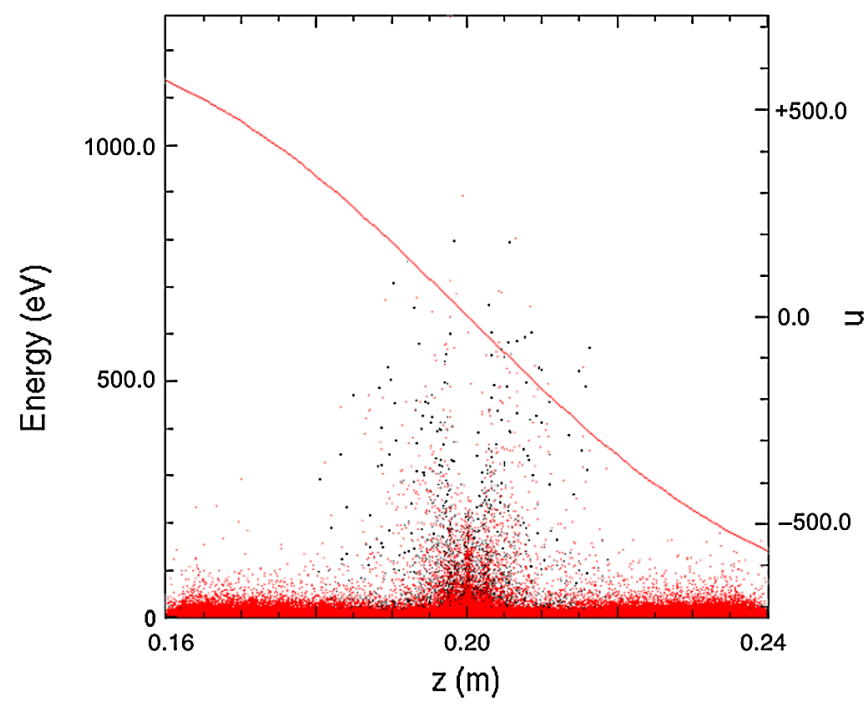

FIG. 15. Energy distribution of macroparticles in a simulation of an $8 \mathrm{~cm}$ range in $z$ centered at a field null, which occurs at $z=0.2 \mathrm{~m}$. Dots are macroparticles. Red dots are secondary macroelectrons; black are primary macroelectrons. The graph is recorded just before the 45th bunch appears, at $616 \mathrm{~ns}$. The curve superimposed is $n$ vs $z$ at $y=0$, where $n$ is the ratio of the cyclotron frequency to the bunch frequency.

tion of the electron density. So what is important in the resonance dynamics, namely, the application of the field of the beam and of the wiggler field to the gyrating electrons, is done correctly in 3D in these simulations, and we would expect to see effects of the resonances, which occur at low magnetic field and where the cyclotron frequency is an integral multiple of the bunch frequency (see Ref. [15]). Figure 15 shows the energy distribution of the macroparticles for a simulation of a short section of the wiggler $(8 \mathrm{~cm})$ centered at the field null. The increase in energy of the electrons near the field null is an indication of the presence of the resonances.

\section{COHERENT TUNE SHIFT CAUSED BY THE ELECTRON CLOUD}

Many calculations of the coherent tune shift in CESRTA have been performed and compared with data [20], with a great deal of success. These calculations were done in 2D using POSINST or ECLOUD [21], and included the drift and dipole regions of the ring. In this paper we present the results of calculations of 3D simulations of the coherent tune shift in the wiggler. As in many of the Cornell calculations, we offset each bunch of the entire bunch train by a given amount ( $2 \mathrm{~mm}$ in the simulations), as was done for most of the experimental tune shift measurements so far. What is required of the simulation in order to calculate the tune shift is to find the electric field gradient due to the electron cloud. The location of the bunch at each $z$ determines the formation of the electron cloud there, so a simulation of the electron cloud buildup must be done for 
any given bunch centroid offset for which we want to know the cloud's electric field at the beam. Therefore, following the procedure devised by Dugan and used in the Cornell simulations [20], in order to find the field gradient experienced by the beam, two electron cloud buildup simulations are done, one with the beam offset by $+2 \mathrm{~mm}$ (e.g., in $y$, for the calculation of the $y$ tune shift) and one with an offset of $-2 \mathrm{~mm}$. The field gradient is calculated assuming the field of the electron cloud is linear in $y$ (or $x$, for the $x$ tune shift). Because we are calculating the coherent tune shift, the field gradient is integrated over the whole bunch. The phase shift for each bunch was calculated, then divided by the beam transit time for one turn to obtain the tune shift in hertz.

The $x$ coherent tune shift in CESR-TA is very small compared to the $y$ tune shift when the measurement is done as outlined above. For a dipole field Dugan has pointed out that the stripe structure of the electron cloud leads to a quadrupole wake that nearly cancels the dipole wake for $x$, causing the $x$ coherent tune shift to be very small. For the $y$ tune shift it required about 40 times as many macroparticles per electron to accurately simulate the tune shift as it did to merely calculate accurate density distributions vs time for the electron cloud, since very good statistics are required, especially for electrons near the beam. This number of macroelectrons per electron was needed at the beginning of the simulation, when the cloud was not near its maximum density, but it was reduced somewhat as the

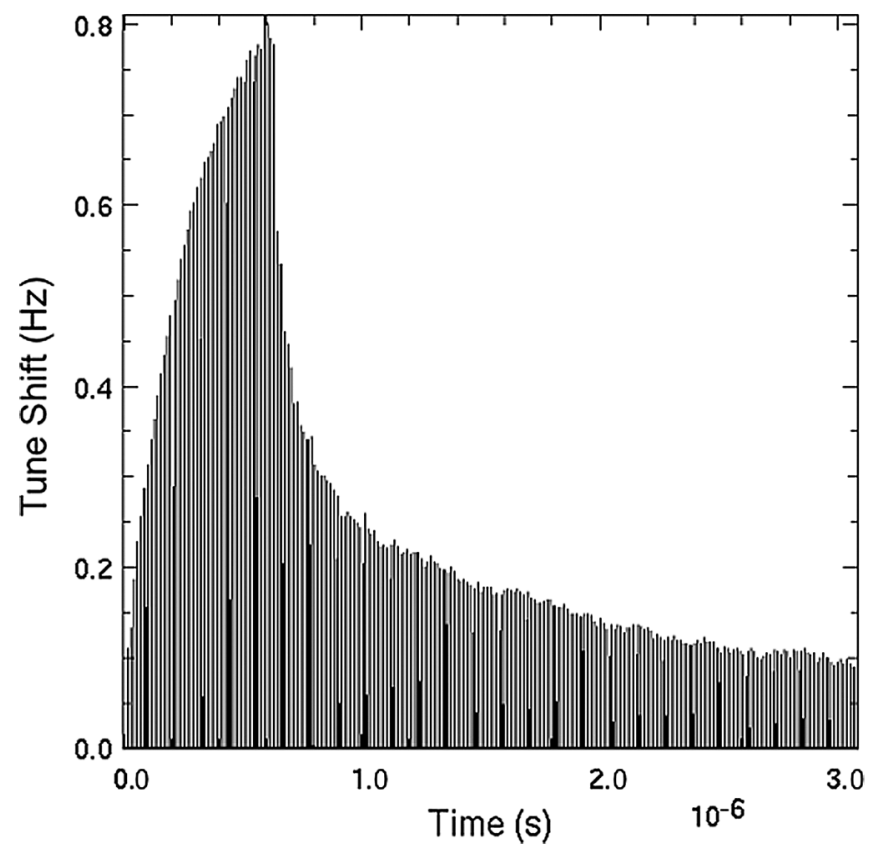

FIG. 16. $y$ coherent tune shift vs time for the beam passage through one wiggler period $(0.4 \mathrm{~m})$ calculated using a 45 -bunch train. The tune shift for each bunch is shown. See text for interpretation beyond bunch 45 . cloud built up. This large number of macroparticles made runs to find the $y$ tune shift very long $(\sim 5$ weeks on 16 processors). Because the $x$ tune shift is so small and its simulation would require many more macroparticles and therefore much longer runs, we have done, and present here, only calculations of the $y$ coherent tune shift. Aside from the beam centroid offsets and number of macroparticles, other parameters were the same as for the runs discussed in Sec. III.

Results are shown in Figs. 16 and 17. Figure 16 shows the $y$ coherent tune shift vs time. Each spike in the graph is the tune shift of a single bunch whose accumulated tune shift, caused by the cloud accumulated and affected by previous bunches, increases as it traverses the wiggler. After the 45th bunch there are no further bunches, and the production of photoelectrons and the electric field of the beam was not present. However, the calculation of the field gradient caused by the electron cloud at the position where an offset bunch would be was calculated anyway with the same spacing in time as the bunch spacing, giving a "tune shift" which is shown in the graph. From this, one can see how the cloud would affect the tune of the first bunch of a subsequent train, depending on the time gap between bunch 45 and the first bunch of that next train. The fact that the tune shift lingers long after the last bunch is due to the longevity of the electrons near the field null.

As a bunch moves through the wiggler, the electrons it encounters at each $z$ location add to the tune shift.

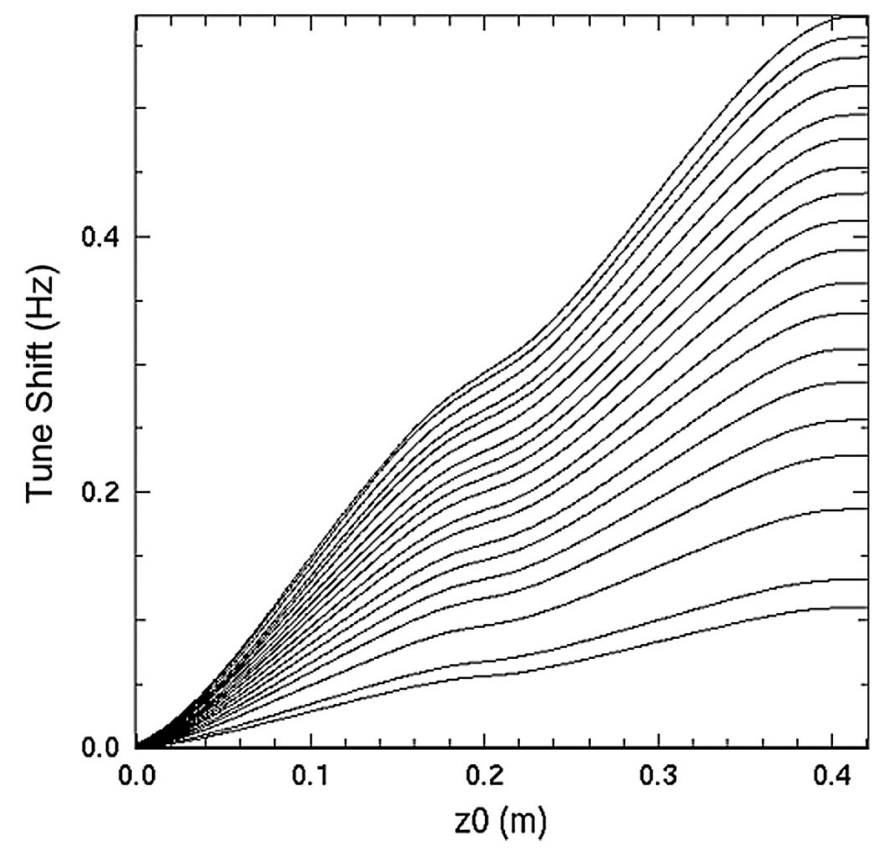

FIG. 17. Tune shift vs position of the center of the beam bunch, z0, for the first 20 bunches of the train (note: contribution of 1 st bunch is very small so on this scale its curve lies along the horizontal axis). The field nulls are at $z=0.0,0.2,0.4 \mathrm{~m}$. Maxima of $B_{y}$ are at $z=0.1,0.3 \mathrm{~m}$. 
Therefore by looking at the accumulated tune shift vs the position of the bunch longitudinal centroid, $\mathrm{z} 0$, one can see the contribution to the total tune shift of different sections of the wiggler. Figure 17 shows the accumulated tune shift versus $\mathrm{z} 0$ for the first 20 bunches. From the slope of the curves in this figure, one can compare the tune shift contributions of different parts of the wiggler. As the cloud builds up with each successive bunch, the slope of the curves near the field null at $z=0.2 \mathrm{~m}$ increases because the $x$ drift of the electrons is bringing them nearer to the beam. By approximately bunch 30 the contribution per centimeter of the electrons near the field null is comparable to the contribution per centimeter of the dipolelike rest of the wiggler. However, because the section near each field null is only approximately $5-6 \mathrm{~cm}$ long, its contribution, even late in the bunch train, is only about a quarter of the total tune shift. The time behavior of the tune shift contributed by the electrons near the field null is of course different from that near the field maxima. As the electrons here drift in $x$ toward the beam at the center of the chamber, their contribution to the tune shift is linear with bunch number through the whole bunch train. The contribution of the electrons near the field maximum, in contrast, has a dependence with bunch number that is only linear for about 5-6 bunches, then begins to bend over, and really begins to saturate around bunch 20 .

The curves of Fig. 17 suggest a method of computing the total tune shift which would save a great deal of computer run time. The tune shift vs z0 curve for any given bunch seems to have one slope for a range of $\mathrm{z} 0$ around the field null and another for all other values of $\mathrm{z} 0$, with a transition between these two slopes that occurs over a very short distance. One could, therefore, simulate short systems, one near the field null and one for $z$ near the $B_{y}$ maximum, to find the tune shift per meter for each bunch in these $z$ ranges. Knowing from one run of a few bunches what the $z$ limits are where each system predicts the tune shift correctly, one could then get a very good estimate for the total tune shift for every bunch from the short system runs.

The CESR-TA experiments have tried to measure a coherent tune shift due to the wiggler in the L0 straight section, but see no measurable difference in the tune shift with the wiggler off vs on. Within experimental uncertainty, this is consistent with the values calculated here. The reason that the wiggler contribution is so small in this experiment is that the reflected photon fluxes in the wiggler are much lower than in the rest of the ring. In addition, the wiggler has a copper vacuum chamber, and thus a much lower secondary emission coefficient, as well as a much shorter length, than the rest of the machine, which has an aluminum vacuum chamber.

In estimating the contribution of the wiggler electron cloud to the coherent tuneshift in other accelerators and rings, it would be helpful to keep in mind that the contribution per unit length of the dipolelike sections of the wiggler would be similar to that of a dipole with the same photon flux at the wall and the same chamber surface. The contribution per unit length of the electron cloud at the field null is similar in magnitude for bunches late in a train to that of the dipolelike sections, but is slower to build up. The speed with which this happens depends on the wiggler magnetic field geometry (its curvature and gradient) near the field null.

\section{SUMMARY AND CONCLUSIONS}

The simulation results show that electron dynamics in the wiggler region is qualitatively different, as expected, in regions near the maxima of the vertical magnetic field versus regions near the vertical field minimum, or "field null", where the longitudinal field magnitude is significant. We have shown the spatial extent of these regions above. Near the field maxima the cloud buildup is similar to what occurs in a dipole, producing two vertical stripes (or a single stripe for lower bunch current) near the $y$ axis. In contrast, near vertical field minima there is a movement of electrons across the chamber in $x$ near the midplane (i.e., $y=0$ ). This motion is caused by the curvature and gradient of the magnetic field, which cause a drift along the $x$ direction of the center of the Larmor orbit. For the parameters used here, the electrons require approximately 45 bunch spacings, or $0.6 \mu \mathrm{s}$, to reach the center of the chamber, traveling in from the two sides to form a horizontal "stripe" with some structure. In general, the drift speed will be dependent on the field magnitude and gradient [see Eq. (1)]. The density of electrons in the horizontal stripe is comparable for the parameters used here to that of the vertical stripes in the dipolelike region (see especially Fig. 10, which shows the density near the beam). Near the end of the bunch train it therefore produces a similar coherent tune shift per centimeter of beam travel. The horizontal stripes are produced by electrons which are born near $y=0$ at the sides of the chamber, while the electrons of the vertical stripes at the dipolelike regions originate at the top and bottom of the chamber near $x=0$. Therefore a change in the distribution of photoelectrons, either due to a change in reflectivity of the chamber surface or due to intervening structures upstream, will change the relative density of the electron clouds in the two regions. For lower photon reflectivity the contribution of the electrons near the field null could exceed that of the electrons in the dipolelike region of the wiggler.

Some evidence of cyclotron resonances is seen near the regions of minimal $B_{y}$, but the simulations discussed here do not have sufficient $z$ resolution to be able to resolve them. It is likely that these resonances enhance the density of the cloud by increasing the energy of the electrons, which in turn results in increased secondary electron production.

Not only the distribution, but also the longevity of the electron cloud is different in these two regions (near 
the field nulls and near the maxima of $B_{y}$ ). The stripes in the dipolelike regions dissipate in a time of the order of $0.6 \mu \mathrm{s}$, while $\sim 45 \%$ of the electrons near the minimum $B_{y}$ regions remain after $2.5 \mu \mathrm{s}$. The long-lived electrons are trapped within the magnetic field, mirroring back and forth in $z$ along field lines. Therefore though these minimum- $B_{y}$ sections of the wigglers are limited in length they may have a disproportionate effect on the beam.

Simulations of multiple 45-bunch trains with 15-bunch gaps between trains show that electrons left from the first train significantly increase the electron cloud density experienced by the second train. However, the train-to-train change in the cloud for subsequent trains (after the second) is much less. With this train spacing some electrons in both sections of the wiggler, dipolelike and near the field null, remain when the next train arrives.

This paper has studied the buildup of the electron cloud but, except for the coherent tune shift caused by the cloud, it has not addressed the effects of that cloud on the beam. The effect of the wiggler electron cloud on beam quality and stability must be determined by self-consistent simulations which follow a beam distribution that evolves in time in response to the effects of the cloud. From the results presented here, however, one can see that the effects from the dipolelike sections of the wiggler will be similar to those in dipoles of similar length and magnetic field strength, and so the effect is straightforward to estimate, given the figures shown above that determine the length of the wiggler where these conditions apply. The effect of the cloud in the rest of the wiggler, where the longitudinal field is significant, remains to be determined. As just described, it has a cloud that is distributed differently spatially from the dipolelike regions, and which builds more slowly near the beam but persists longer. The tune shift results above give an indication that its effect on the coherent tune shift might be $\sim 1 / 3$ that of the dipolelike regions during the passage of a bunch train. Nevertheless, its longer-term results remain to be investigated.

\section{ACKNOWLEDGMENTS}

The author would like to acknowledge insightful suggestions from Gregory Penn of Lawrence Berkeley National Laboratory concerning the movement of electrons near the field null. Gerald Dugan (Cornell University) was extremely helpful in explaining the tune shift calculation method which best models the CESR-TA "pinged-beam" measurement. The author would like to thank Mark Palmer and the members of the CESR-TA group at Cornell and the electron cloud group in the Center for Beam Physics at LBNL for feedback and suggestions during the course of this work and for their fine collaborative spirit. Finally, this paper would not have been possible without coding and support from David Grote and Jean Luc Vay, developers of the WARP code, who gave generously of their time and skill. This work was supported by the National Science Foundation under Contract No. PHY-0734867 and the Office of Science, U.S. Department of Energy, under Contract No. DE-FC0208ER41538.

[1] The topic has been the subject of a series of dedicated workshops, the most recent one being ECLOUD07, Proceedings of the International Workshop on ElectronCloud Effects "ECLOUD07", edited by H. Fukuma, E. S. Kim, and K. Ohmi, KEK Proc. No. 2007-10 (Daegu, South Korea, 2007), http://chep.knu.ac.kr/ecloud07/.

[2] W. M. Sharp et al., in Proceedings of the 2007 Particle Accelerator Conference, Albuquerque, New Mexico (IEEE, Albuquerque, New Mexico, 2007), http:// accelconf.web.cern.ch/AccelConf/p07/PAPERS/

THPAS050.PDF. See also the newsletter: ICFA Beam Dynamics Newsletter 48 (2009), especially papers by Drago (pp. 50-58), Demma (pp. 64-71), Ng and Pivi (pp. 105-111), and Fukuma (pp. 112-116).

[3] M. Pivi, in Proceedings of ECLOUD04, 2005, p. 155, http://mafurman.lbl.gov/ECLOUD04_proceedings/pivifutureLCs-v6.pdf.

[4] D. Schulte, R. Wanzenberg, and F. Zimmermann, in Proceedings of ECLOUD04, 2005, p. 183, http:// mafurman.lbl.gov/ECLOUD04_proceedings/wanzenbergtesla_clic_dr-v3.pdf .

[5] F. Zimmermann et al., Report No. EUROTeVReport_2006_002-1, CLIC Note 650.

[6] M. Pivi and T. Raubenheimer, in Proceedings of the 20th Particle Accelerator Conference, Portland, OR, 2003 (IEEE, New York, 2003).

[7] C. M. Celata et al., in Proceedings of ECLOUD07, 2007 (Ref. [1]), p. 104.

[8] W. Bruns, D. Schulte, and F. Zimmermann, in Proceedings of the 2007 Particle Accelerator Conference, Albuquerque, New Mexico (Ref. [2]), p. 3375.

[9] L. Wang and F. Zimmermann, in Proceedings of the 2007 Particle Accelerator Conference, Albuquerque, New Mexico (Ref. [2]), p. 1808.

[10] L. Wang, X. Huang, and M. Pivi, in Proceedings of the 2010 International Particle Accelerator Conference (Kyoto, Japan, 2010), p. 1892, http://accelconf.web .cern.ch/AccelConf/IPAC10/papers/tupec077.pdf.

[11] J.-L. Vay, C. M. Celata, M. Furman, M. Venturini, and K. G. Sonnad, in Proceedings of the 23rd Particle Accelerator Conference, Vancouver, Canada, 2009 (IEEE, Piscataway, NJ, 2009); D. P. Grote, A. Friedman, J.-L. Vay, and I. Haber, AIP Conf. Proc. 749, 55 (2005).

[12] M. A. Furman and G. R. Lambertson, in Proceedings of the International Workshop on Multibunch Instabilities in Future Electron and Positron Accelerators "MBI-97", edited by Y.H. Chin, KEK Proc. No. 97-17 (KEK, Tsukuba, Japan, 1997), http://www-acc.kek.jp/ WWW-ACC-exp/Conferences/MBI97-N/MBI97.html

[13] M. A. Furman, LBNL-41482/CBP Note 247/CERN LHC Project Report No. 180, 1998. 
[14] M. A. Furman and M. T.F. Pivi, Phys. Rev. ST Accel. Beams 5, 124404 (2002).

[15] C. M. Celata, Miguel A. Furman, J.-L. Vay, and Jennifer W. Yu, Phys. Rev. ST Accel. Beams 11, 091002 (2008).

[16] F. Zimmermann, Report No. SLAC-PUB-7425, LHC Project Report 95, 1997.

[17] M. A. Furman and G. R. Lambertson, in Proceedings of EPAC96 (Report No. LBNL-38220/PEP-II AP 96-03/CBP Note-173, 1996).

[18] M. A. Furman and G. R. Lambertson, in Proceedings of the Particle Accelerator Conference, Vancouver, BC, Canada, 1997 (IEEE, New York, 1997).
[19] J.R. Calvey et al., in Proceedings of the 23rd Particle Accelerator Conference, Vancouver, Canada, 2009 (Ref. [11]); J. Calvey et al., in Proceedings of the 2010 International Particle Accelerator Conference (Ref. [10]), TUPD022.

[20] J. A. Crittenden et al., in Proceedings of the 23rd Particle Accelerator Conference, Vancouver, Canada, 2009 (Ref. [11]); J. Crittenden et al., in Proceedings of the 2010 International Particle Accelerator Conference (Ref. [10]), TUPD024.

[21] G. Rumolo and F. Zimmermann, Report No. CERN-SLNote-2002-016-AP, 2002. 